19

\title{
The Effect of Morphologies of Embedded Plasmonic Cu-nanoparticles on Solar Absorption of Perovskite Solar Cells: A Comprehensive Study*
}

\author{
(C) Shreya Sahai ${ }^{1}$, Anshu Varshney ${ }^{2}$ \\ ${ }^{1}$ Department of Electronics and Communication, \\ JIT, Noida 201307, India \\ ${ }^{2}$ Department of Physics and Material Science and Engineering, \\ JIIT Noida 201307, India \\ e-mail: anshu.varshney@jiit.ac.in
}

Received March 20, 2021

Revised April 20, 2021

Accepted April 23, 2021

In this work, we aim to reduce the production costs of Perovskite solar cells (PSCs) and achieve high solar absorbance with the inclusion of different shapes of plasmonic nanoparticles within its $200 \mathrm{~nm}$ thick Perovskite layer. We have performed an extensive study based on the different morphologies of plasmonic nanoparticles including nanospheres, nanocubes, nanocylinders, nanorods, nanotriangular plates embedded within the film. This geometric study is extended to the different orientations of a particle within the film, with respect to the source of light. Based on our previous study involving different materials of nanoparticles within the perovskite film, copper is selected as a potential candidate to drastically reduce the production costs without compromising the absorption efficiency of the cell. Varying sizes of these copper nanoparticles are placed at the centre of the film to verify the enhancement of the light trapping efficiency of these designed cells. We find that the absorption efficiency of PSCs highly depends upon the corner sharpness and orientation of a nanoparticle within the film, though the average absorption remains analogous to the spherical particles. Since the designed PSC with tailored nanoparticles portray a significant variation in its absorbance efficiency with the change in its geometrical parameters, hence we can choose a specific morphology of the particle, placed at a certain angle within the film to obtain maximum absorbance. The entire study is based on the Finite Difference Time Domain (FDTD) method of simulation.

Keywords Perovskite solar cells, solar absorbance, plasmonic Nanoparticles, FDTD 